\title{
Comparison of the GUM and Monte Carlo measurement uncertainty techniques with application to effective area determination in pressure standards
}

\author{
V. Ramnath^ \\ NMISA, Private Bag X34, Lynnwood Ridge, 0040, South Africa
}

Received: 15 November 2009 / Accepted: 5 February 2010

\begin{abstract}
A common measurement model for a gas operated piston-cylinder based pressure standard effective area is the well known integral equation formulation originally developed by Dadson of the NPL. However a problem with directly applying this exact mathematical model is that it cannot be easily cast into a functional form suitable for application of the Guide to the expression of Uncertainty in Measurement (GUM) which is reliant on the concept of sensitivity coefficients without various simplifications. In this paper, we examine the standard approximations that are currently necessary in order to directly apply the GUM for a pressure standard effective area uncertainty determination. We also compare and contrast this to the exact effective area uncertainty results obtained through the direct application of the Monte Carlo Method (MCM) which has recently been published as Supplement 1 to the GUM. Based on these investigations we also draw some preliminary conclusions on the relative merits on the extent to which the shape of the piston and cylinder radii and whose uncertainties may vary along the engagement length of the piston-cylinder may be modeled and incorporated into a piston-cylinder's effective area uncertainty calculation.
\end{abstract}

Keywords: Pressure; effective area; GUM; Monte Carlo

\section{Introduction}

The main body of work for the piston-cylinder based pressure balance's theory is that of Dadson et al. [1] who initiated much of the original research into piston-cylinder assemblies for pressure metrology based on certain simplifications originally considered by Peggs [2]. Subsequent work such as Samaan's [3] has expanded and built on these earlier works using more modern numerical techniques. While the theory of gas operated pressure balances can be considered as a mature and relatively complete field, uncertainty analysis techniques continue to be refined, developed and applied in new ways. This paper will examine the standard approximations that are currently used in pressure balance uncertainty calculations to apply the GUM [4] and compare and contrast these approximate uncertainty results with exact uncertainty results that are obtained through the direct application of the Monte Carlo Method (MCM) as recommended in GUM Supplement 1 [5].

\section{Review of effective area measurand model formulations}

Within the framework of the GUM is the prerequisite of a mathematical model of the measurand which in general

\footnotetext{
^VRamnath@nmisa.org
}

can consist of univariate/multivariate, implicit/explicit and real/complex characteristics as described by Cox and Harris [6] for which effective area $S$ has the exact defining equation

$$
S=\pi r_{0}^{2}\left[1+\frac{h_{0}}{r_{0}}-\frac{1}{r_{0}\left(p_{1}-p_{2}\right)} \int_{0}^{l}(u+U) \frac{d p}{d x} d x\right]
$$

where the pressure distribution in the interface gap for a compressible viscous fluid such as in a gas operated pressure balance is:

$$
\begin{aligned}
p= & {\left[p_{1}^{2}-\left(p_{1}^{2}-p_{2}^{2}\right) \frac{\int_{0}^{x} h^{-3} d x}{\int_{0}^{l} h^{3} d x}\right]^{1 / 2} } \\
\frac{d p}{d x}= & -\frac{1}{2} \frac{p_{1}^{2}-p_{2}^{2}}{h^{3}} \\
& \times\left[\int_{0}^{l} h^{-3} d x\left\{p_{1}^{2}-\left(p_{1}^{2}-p_{2}^{2}\right) \frac{\int_{0}^{x} h^{-3} d x}{\int_{0}^{l} h^{-3} d x}\right\}^{1 / 2}\right]^{-1}
\end{aligned}
$$

where $l$ is the engagement length of the piston cylinder with $0 \leq x \leq l, r(x)$ and $R(x)$ represent the instantaneous piston and cylinder radii along the engagement length, $r_{0}=r(0), R_{0}=R(0), p_{1}$ and $p_{2}$ are the pressures at the bottom and top of the piston-cylinder unit. The formulas $u(x)=r(x)-r_{0}$ and $U(x)=R(x)-R_{0}$ are the fluctuations in the piston and cylinder profiles from the radii values of 
the piston and cylinder at the bottom of the engagement noting that $r_{0}+h_{0}=R_{0}$.

In the case of compressible fluids such as in gas operated pressure balances for non-simple geometries (1) must be solved numerically. Although $S$ is only a function of the dimensional profile of the interface gap between the piston and cylinder and the ratio of pressures $p_{1} / p_{2}$ in the asymptotic limit as $p_{1} \gg p_{2}$ i.e. the 'absolute' mode as $p_{2} \rightarrow 0$ Dadson et al. [1] calculates a purely geometric formula

$$
\begin{aligned}
S^{*}= & \pi r_{0}^{2}\left[1+\frac{h_{0}}{r_{0}}+\frac{1}{r_{0}} \int_{0}^{l} \frac{d(u+U)}{d x}\right. \\
& \left.\times\left\{1-\int_{0}^{x} h^{-3} d x / \int_{0}^{l} h^{-3} d x\right\}^{1 / 2} d x\right] .
\end{aligned}
$$

Previous work by Buonanno et al. [7] in applying the GUM to estimate uncertainty relies on writing the effective area (1) as

$$
A_{0}=\pi r_{0}^{2}\left\{1+\frac{h_{0}}{r_{0}}+\frac{1}{r_{0}} \frac{\int_{0}^{l} \frac{u+U}{h^{3}} d z}{\int_{0}^{l} h^{-3} d z}\right\} .
$$

This is an equivalent form of the effective area in the asymptotic limit $p_{1}-p_{2} \ll p_{1}$ i.e. zero applied pressure so that $A_{0}$ is only a function of $\mathbf{x}=\left[x_{1}, \ldots, x_{n}\right]$, $\mathbf{r}=\left[r_{1}, \ldots, r_{n}\right]$ and $\mathbf{R}=\left[R_{1}, \ldots R_{n}\right]$ where $n$ is the number of diameter measurements along the engagement length. Estimating the uncertainty of effective area using the GUM methodology Buonanno et al. [7] found an approximate formulation for the standard uncertainty as

$$
\begin{aligned}
u^{2}\left(A_{0}\right)= & \left(\frac{\partial A_{0}}{\partial r_{0}}\right)^{2} u^{2}\left(r_{0}\right)+\left(\frac{\partial A_{0}}{\partial r}\right)^{2} u^{2}(r) \\
& +\left(\frac{\partial A_{0}}{\partial R}\right)^{2} u^{2}(R)+2 \frac{\partial A_{0}}{\partial r_{0}} \frac{\partial A_{0}}{\partial r} u\left(r_{0}\right) u(r) \rho\left(r_{0}, r\right) \\
& +2 \frac{\partial A_{0}}{\partial r_{0}} \frac{\partial A_{0}}{\partial R} u\left(r_{0}\right) u(R) \rho\left(r_{0}, R\right)
\end{aligned}
$$

where $\rho\left(x_{i}, x_{j}\right)$ represents the correlation coefficient between the inputs $x_{i}$ and $x_{j}$ of the measurand model with the following sensitivity coefficients:

$$
\begin{aligned}
& \frac{\partial A_{0}}{\partial r_{0}}=\frac{A_{0}}{r_{0}}-\pi r_{0} \\
& \frac{\partial A_{0}}{\partial r}=\pi r_{0}\left[\int_{0}^{l} \frac{(4 R+2 r)}{(R-r)^{4}} d z \int_{0}^{l} \frac{1}{(R-r)^{3}} d z\right. \\
& \left.\quad-\int_{0}^{l} \frac{r+R}{(R-r)^{3}} d z \int_{0}^{l} \frac{3}{(R-r)^{4}} d z\right]\left[\int_{0}^{l} \frac{1}{(R-r)^{3}} d z\right]^{-2}
\end{aligned}
$$$$
\begin{aligned}
& \frac{\partial A_{0}}{\partial R}=\pi r_{0}\left[\int_{0}^{l} \frac{(-2 R-4 r)}{(R-r)^{4}} d z \int_{0}^{l} \frac{1}{(R-r)^{3}} d z\right. \\
& \left.-\int_{0}^{l} \frac{r+R}{(R-r)^{3}} d z \int_{0}^{l} \frac{-3}{(R-r)^{4}} d z\right]\left[\int_{0}^{l} \frac{1}{(R-r)^{3}}\right]^{-2}
\end{aligned}
$$

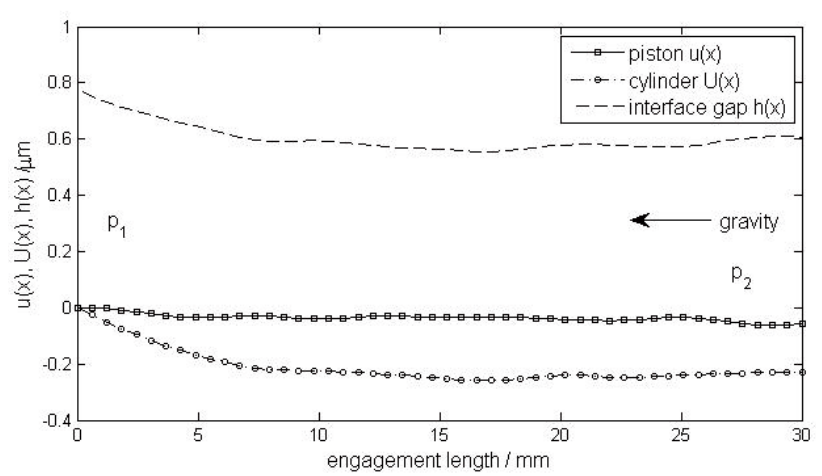

Fig. 1. Piston and cylinder fluctuations for a DHI pressure balance with $h_{0}=0.779 \mu \mathrm{m}$ and $l=30 \mathrm{~mm}$.

Equation (3) although based on an analysis for an incompressible fluid can be used as a general measurand model for effective area uncertainty calculations even in gas operated pressure balances where the fluid is compressible since Dadson et al. [1] proved that the formula for a compressible fluid in the limit $p_{1}-p_{2} \ll p_{1}$ of zero applied pressure effective area to be equivalent to that of an incompressible fluid. As a result this will be used as the general measurand model for a pressure balance's effective area in this paper that can be analyzed using both the GUM and MCM techniques in order to perform a useful comparison.

To quantify the differences in results using these formulae representative geometry data of the pressure balance in Figure 1 with a sequence of applied pressures $\left(p_{1}-p_{2}\right)$ were used and calculations performed in Matlab using the available dimensional data in Figure 1. These calculations yielded an extrapolated value to zero applied pressure of $S_{0}=1961.061318 \mathrm{~mm}^{2}$ for the exact formula, $A_{0}=1961.062218 \mathrm{~mm}^{2}$ for the approximate zero applied pressure formula and $S^{*}=1961.061224 \mathrm{~mm}^{2}$ the asymptotic absolute pressure formula using a trapezoidal integration algorithm trapz for fast computations applied to a spline fit of the integration data made up of 500 integration data points, which indicate that formulae for an effective area determined solely through dimensional measurements are generally accurate to at least $\pm 0.5 \mathrm{ppm}$.

For the general measurand model numerical integrations of terms like $x$ and $h^{-1 / 3}$ are necessary. This can be performed natively within Matlab either by utilizing high accuracy routines like the adaptive Simpson and Lobatto quadrature routines which take a longer time to converge or alternately with the simpler, quicker but lower accuracy trapezoidal integration routine available. A comparison of routines available indicates that a simple trapezoidal integration is accurate to $\pm 0.005 \mathrm{ppm}$. As a result computations are performed with a trapezoidal integration algorithm due to the combination of fast computational speed necessary for the practical implementation of the MCM uncertainty technique and the relatively small numerical errors.

Thus numerical errors introduced through the choice of integration algorithms and round off errors in the implementation of the models present will not be explicitly 
considered in this paper as the focus will be on a comparison of the results of the GUM and MCM techniques on the same measurand model.

\section{Investigation into uncertainty analysis using the GUM and MCM}

The application of the GUM in any uncertainty analysis is based on three main assumptions, namely (i) the non-linearity of a measurand as modeled by a function $f(\mathbf{X})$ must be insignificant (Clause 5.1.2), (ii) the Central Limit Theorem (CLT) must be assumed for the model of the measurand (Clauses G.2.1 and G.6.6), and (iii) the necessary conditions for the Welch-Satterthwaite (W-S) formula to calculate the effective degrees of freedom $\nu_{\text {eff }}$ must apply (Clause G.4.2).

In addition to the GUM Supplement 1 we can add an additional requirement (iv) that the calculation of the partial derivatives of the model must not be unnecessarily difficult or inconvenient to compute.

Similarly the application of the MCM is based on five main assumptions, namely (a) $f(\mathbf{X})$ is continuous with respect to elements $X_{i} \in \mathbf{X}$ where the probability density function (PDF) of input $X_{i}$ is $g_{i}\left(\xi_{i}\right)$, (b) the distribution function $G(\eta)=\int_{-\infty}^{\eta} g(z) d z$ for $Y=f(\mathbf{X})$ is continuous and strictly increasing, (c1) the PDF $g(\eta)$ for $Y$ is continuous over the interval for which $g(\eta)$ is strictly positive, (c2) $g(\eta)$ is unimodal, (c3) $g(\eta)$ is strictly increasing/zero to the left of the mode and strictly decreasing/zero to the right of the mode, (d) the expectation of $Y$ i.e. $E(Y)$ and variance of $Y$ i.e. $V(Y)$ exists, and (e) a sufficiently large number of Monte Carlo events $M$ is utilized in the simulation.

We can write $(3)$ as a function $A_{0}=f(\mathbf{X})$ with the vector $\mathbf{X}=[\mathbf{x}, \mathbf{r}, \mathbf{R}]$ considered as inputs of the model and which completely define $A_{0}$ and thereby classify the model as being real, univariate and explicit following the guidelines of Cox and Harris [6]. These authors distinguish between an implicit and explicit model through the availability of the measurand to be represented by means of a readily/stably explicit representation, of which the integration process is well known to be numerically stable and to "smooth" noisy data e.g. derivative terms in the integrand. In addition final results can be explicitly represented through the use of schemes such as Simpson's integration as discussed by Burden and Faires [8].

With reference to the four GUM conditions we can immediately confirm that condition (ii) which is the validity of the CLT applies due to the large number of dimensional data points which for a dimensional measurement of a pressure balance is typically with $n=25$ then $25 \times 3=75$ inputs. Condition (i) would in general not be satisfied due to the relatively large sensitivity of the effective area at the required accuracy levels of order of $10 \mathrm{ppm}$ with the piston-cylinder interface gap of order $0.5 \rightarrow 1 \mu \mathrm{m}$ as can be seen from the $h(x)$ values of a pressure balance in Figure 1.

Condition (iii) which concerns the validity of the $\mathrm{W}$-S formula is only applicable when the components of the measurement error with finite degrees of freedom are uncorrelated where the validity of the W-S formula is necessary in order to calculate $\nu_{\text {eff }}$ to convert the measurand's standard uncertainty into its expanded uncertainty e.g. at 95\% confidence level. Willink [9] has recently proposed a generalization to the W-S formula in the case of correlated inputs of the form

$$
\begin{aligned}
\nu_{\text {eff }}^{\prime} & =\frac{u_{y}^{4}}{\sum_{i=1}^{n}\left(\sum_{j=1}^{n} c_{i} c_{j} r_{i j} u_{i} u_{j}\right)^{2} / \nu_{i}} \\
\nu_{\text {gen }} & =\max \left\{1, \nu_{\text {eff }}^{\prime}\right\}
\end{aligned}
$$

where $c_{i}$ is the is the sensitivity coefficient $\partial f / \partial x_{i}$ for input $x_{i}$ and $r_{i j}=\rho\left(x_{i}, x_{j}\right)$ is the corresponding correlation coefficient of inputs $x_{i}$ and $x_{j}$ which we will investigate in this paper for the six possible correlation terms as a modification within the framework of the GUM in order to test for the confidence interval of the measurand corresponding to its expanded uncertainty against the results of a MCM calculation.

In this paper our approach in comparing the results of the effective area uncertainty with the GUM and MCM techniques will therefore focus on the differences of the measurand's final uncertainty based on the differences of how both methods incorporate the sensitivity coefficients and correlation effects.

\section{Results and comparison of different effective area formulations}

We performed computations to study the effective area uncertainty results using the dimensional data inputs in Figure 1 where the corresponding dimensional standard uncertainties were taken as $u(r)=0.25 \times$ $10^{-6} \sqrt{(0.07)^{2}+(4 r)^{2}}$, where $r$ is the piston radius in meters, $u(R)$ the cylinder radius uncertainty has the same formula, and $u(x)=0.5 \times 10^{-6} \sqrt{0.01+4 x^{2}}$ is the uncertainty along the engagement length with $x$ measured in meters.

The derivation of formula (3) by Buonanno et al. [7] is based on the application of the Leibniz integral rule which correctly calculates terms $\partial A_{0} / \partial r$ and $\partial A_{0} / \partial R$ that are constant but incorrectly utilized as sensitivity coefficients. This is due to the fact that $r=r(z)$ and $R=R(z)$ in (3) i.e. the radii are not single input quantities for the model but vector quantities varying along the engagement length and as a result do not have a single value.

Intuitively we expect the representative values of $r$ and $R$ used in (3) to be close to either the arithmetic means or a weighted average of the respective radii by recalling that a simple measurand model relying only on a piston and cylinder radii is $A_{0}^{\|}=\frac{\pi}{2}\left[\langle r\rangle^{2}+\langle R\rangle^{2}\right]$ where $\langle\bullet\rangle$ signifies an arithmetic mean of the available data. Unfortunately the form for $A_{0}$ in terms of $r_{0}$, and the single representative values of $r$ and $R$ to use in the uncertainty calculation is not explicit. We must therefore assume weighted averages of radii values of the form 


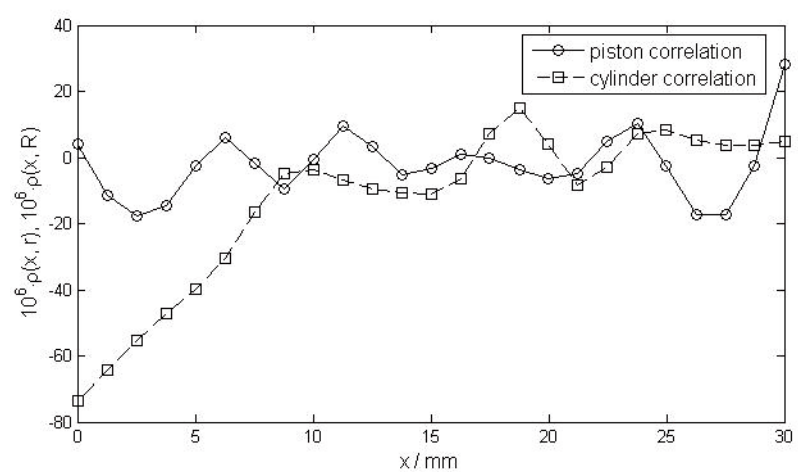

Fig. 2. Approximate correlation coefficients between engagement length and piston/cylinder radii.

$y=\sum_{i=1}^{n}\left(x_{i} / u^{2}\left(x_{i}\right)\right) / \sum_{i=1}^{n}\left(1 / u\left(x_{i}\right)^{2}\right)$ with an associated standard deviation of this reference value calculated as $1 / u^{2}(y)=\sum_{i=1}^{n}\left(1 / u\left(x_{i}\right)^{2}\right)$ in the absence of further information.

Before being able to perform the GUM and MCM uncertainty analyses we must first specify the correlation effects between the inputs of the measurand which in the case of the GUM framework must be specified by correlation coefficients and which in the case of the MCM framework must be specified through a mathematical relationship between the inputs which may be either explicit or implicit. As a proposed estimate for the correlation coefficient $\rho\left(x_{i}, x_{j}\right)$ between inputs $x_{i}$ and $x_{j}$ we have from the GUM the approximation $\rho\left(x_{i}, x_{j}\right) \approx\left[u\left(x_{i}\right) \delta_{j}\right] /\left[u\left(x_{j}\right) \delta_{i}\right]$ (Appendix C.3.6 in the GUM for experimental determination of correlation coefficients) where $\delta_{i}$ is a disturbance in $x_{i}$ that results in a disturbance $\delta_{j}$ in $x_{j}$.

It should be noted that according to this formulation whilst correlations $\rho\left(x_{i}, r_{i}\right)$ and $\rho\left(x_{i}, R_{i}\right)$ do exist as indicated in Figure 2 using the approximate formula for correlations and the mathematical representations for the radii, then mathematically no correlation between the piston radii and the cylinder radii since the piston and cylinder measurements are considered to be independent functions of the distance along the engagement length (although they may be measured using the same dimensional equipment).

Physically even if measurements are taken with the same equipment no mechanism or mathematical model presently exists with sufficient detail to uniquely infer how the action of taking measurements of piston diameters will influence the measurement taking of cylinder diameters and vice versa. Multiple calibrations and repeated measurements by different calibration metrologists further reduce the likelihood of such a correlation.

Thus currently there is no obvious compelling mathematical or physical reason to suppose that correlation effects between the piston and cylinder diameters are significant and recent investigations such as in the EUROMET 740 project Molinar et al. [10] demonstrate different opinions on whether the correlation effects $\rho(r, R)$ are non-zero or significant enough to be included. In model (4) there is then subjectivity in $\rho(r, R)$ and in addition a requirement that $\rho\left(r_{0}, r\right)$ and $\rho\left(r_{0}, R\right)$ be specified.
Consequently model (4) (although contradictory due to the combination of 'sensitivity coefficients' calculated with Eq. (3) for the approximate effective area $A_{0}$ for varying radii geometry profiles and single reference uncertainty values for $r$ and $R$ from the straight concentric piston and cylinder radii model $A_{0}^{\|}$) is able to offer an effective area uncertainty estimate if we choose to calculate the nominal piston and cylinder radii as weighted averages using the standard formula. The multiple piston/cylinder radii along the engagement length can then be interpreted as single representative values by setting $\langle r\rangle=\sum_{i=1}^{n}\left(\left(r_{i}\right) / u^{2}\left(r_{i}\right)\right) / \sum_{i=1}^{n}\left(1 / u^{2}\left(r_{i}\right)\right)$ and similarly for $\langle R\rangle$.

This approach when adopted appears to be the only mechanism to calculate as opposed to estimating the correlation between the piston and cylinder radii since in this case the model defined by $A_{0}^{\|}$allows us to compute the covariance between $r$ and $R$ as $\rho(\bar{r}, \bar{R})=$ $\left[\sum_{i=1}^{n}\left(r_{i}-\bar{r}\right)\left(R_{i}-\bar{R}\right)\right] / n(n-1)$. This formula, when calculated with experimental data with a finite number of experimental dimensional measurements is $\rho(\bar{r}, \bar{R})=$ $6.931213 \times 10^{-17}$ suggesting that radii correlation effects are generally negligible.

To calculate $u\left(A_{0}\right)$ using the GUM formulation in (4) we must also specify $\rho\left(r_{0}, r\right)$ and $\rho\left(r_{0}, R\right)$ which we calculate using the approximation specified in the GUM as $\rho\left(r_{0}, r\right)=\left[u\left(r_{0}\right) \delta_{r}\right] /\left[u(r) \delta_{r_{0}}\right]=0.076923$ and $\rho\left(r_{0}, R\right)=$ $\left[u\left(r_{0}\right) \delta_{R}\right] /\left[u(R) \delta_{r_{0}}\right]=0$ in the absence of a ready means to adequately describe any correlation between $r$ and $R$. Substitution of these values then leads to standard uncertainties in the cases for the zero correlations, calculated correlations, and unity correlations of $\pm 1.726804 \mathrm{ppm}$, $\pm 1.726806 \mathrm{ppm}$ and $\pm 2.441831 \mathrm{ppm}$ respectively.

As a result, when calculating the effective area using a GUM formulation we conclude that the three correlation effects in the existing GUM uncertainty formula for $u\left(A_{0}\right)$ are unlikely to contribute any significant changes and should only be retained, if at all, as control inputs to adjust the calculated uncertainty in order to bring the GUM value closer to the exact value obtained with a MCM uncertainty analysis. The radii values $r$ and $R$ should be modified to incorporate the shape of the piston and cylinder.

The exact value of $u\left(A_{0}\right)$ for $A_{0}(\mathbf{x}, \mathbf{r}, \mathbf{R})$ was determined with the MCM technique using $50 \times 10^{3}$ Monte Carlo simulation events following the approach of Cox and Harris [11] to implement the MCM for the measurand model in equation (3). Computations were performed in Matlab and the method utilized is similar to the ideas presented in earlier work performed by Cordero et al. [12] for the experimental integral of a UV index however we utilize the formal methodology of the MCM with two differences in our implementation. The first is the distinction between the model inputs and known parameters which reduces the computational cost as it avoids the use of a Dirac- $\delta$ PDF which are exactly known, and the second is the utilization of more accurate statistical formulae for the measurand in terms of the underlying distribution function $G(\eta)$. As discussed by Cordero et al. [12] the 

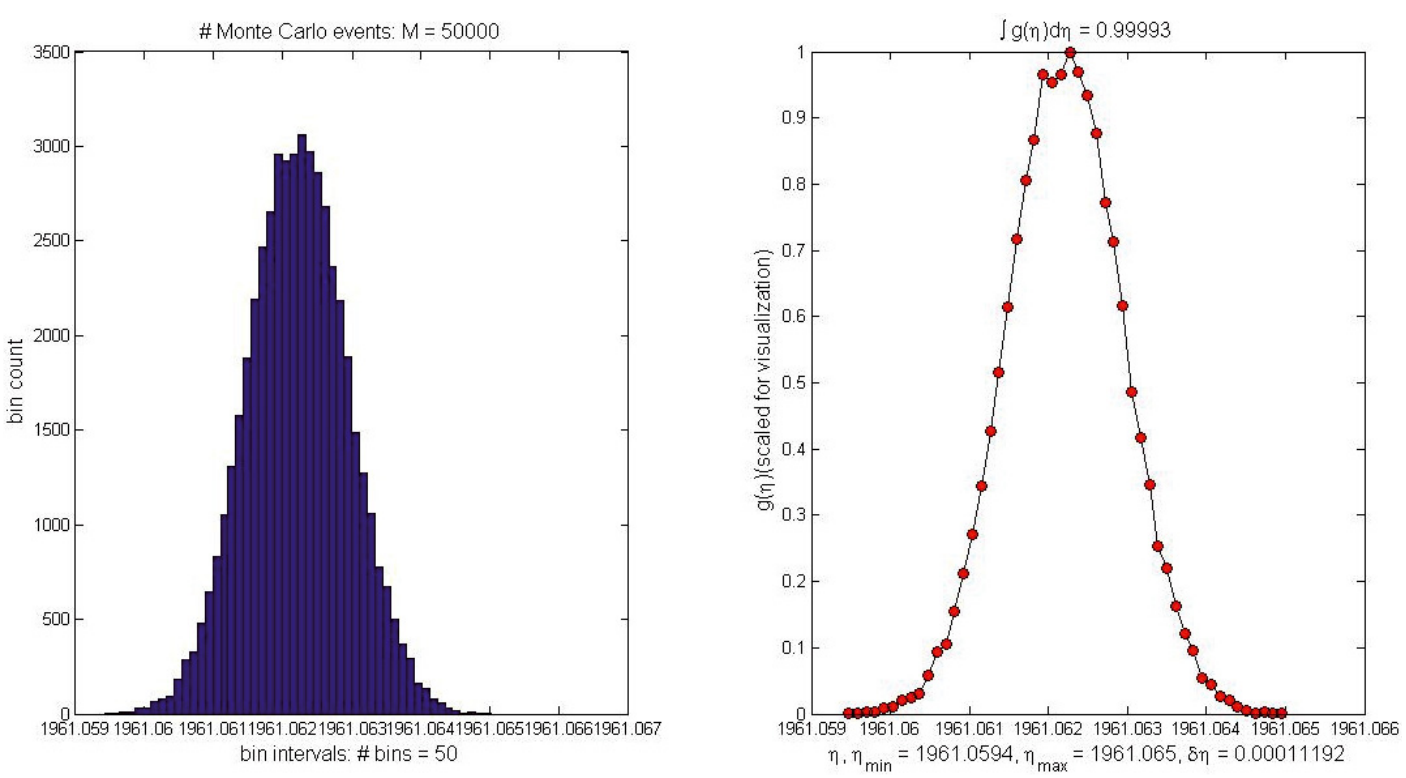

Fig. 3. Histogram and scaled probability density function for Monte Carlo method uncertainty analysis.

model $M$ for the measurand $Q=M(\mathbf{P})$ where the input $\mathbf{P}=\left[x_{1}, \ldots, x_{J}, y_{1}, \ldots, y_{J}\right]^{T}$ has a spatial resolution e.g. $(\Delta x, \Delta y)$ in the case $Q=\int_{a}^{b} y(x) d x$ and a spatial period i.e. the length $J$ of $\mathbf{P}$ determines the accuracy of $Q$. It should be noted that the interpolating function used is determined in accordance with physical, numerical or statistical criteria.

As per the observation by Cordero et al. [12] that an inadequate spatial resolution can lead to systematic errors in the model input which in our case would correspond to a small number of piston/cylinder diameter measurements along the engagement length we also remark that an artificial bias can also be introduced if the spatial resolution is artificially increased through interpolation. Whilst Cordero et al. [12] propose additional input quantities (corrections) to counter systematic errors introduced through inadequate spatial resolution which must also be considered in the uncertainty propagation (because the corrections introduced actually alter the underlying mathematical model) leading to a greater output uncertainty, we recommend instead that the interpolation function only utilizes existing physical experimental data in constructing the interpolation model.

Sampling from the inputs PDF's utilized the built in Matlab random number generators which in the case of a Gaussian PDF $N\left(\mu, \sigma^{2}\right)$ was implemented in form $\mu+\sigma z$ with $z=\operatorname{randn}()$, and in the case of a rectangular PDF in the interval $(a, b)$ of form $a+(b-a) r$ with $r=\operatorname{rand}()$.

This approach was adopted in preference to the use of a Wichmann-Hill random number generator (RNG) algorithm and Box-Muller Gaussian pseudo-random number generator as recommended in Appendix C.3 and C.4 of the MCM for simplicity. The main Matlab RNG based on a Mersenne Twister algorithm has already been extensively tested/validated, is readily available and in addition is particularly suitable for Monte Carlo simulations since it has a long period.
The inputs of positions along the piston/cylinder engagement length are considered as independent, whilst the corresponding piston and cylinder radii measurements $r_{i}$ and $R_{i}$ are considered as functions of $x_{i}$ as previously discussed. In order to perform an accurate estimate for $A_{0}$ as per the final recommendations of Molinar et al. [10] it is beneficial for a large number of piston/cylinder diameters to be available, however it should be noted that in order to perform a realistic uncertainty estimate for $A_{0}$ we should limit the input dimensional data in the MCM analysis to measurements that have been experimentally performed as an excessive number of interpolated i.e. inferred diameter data will lead to an artificially low value for $u\left(A_{0}\right)$. This precaution can be understood as not inferring additional information that is not possible to deduce within the limits imposed by the existing mathematical model of the measurand. Due to the fact that the dimensional characterization of the piston/cylinder profiles were performed in-house with corresponding measurement uncertainties estimated in accordance with the GUM, all the inputs were considered to have Gaussian PDF's where the expected values $\mu_{i}$ for $x_{i}$ were taken as the nominal values in the calibration certificates and the variance was specified as the corresponding uncertainty i.e. $\sigma_{i}^{2}=u^{2}\left(x_{i}\right)$.

The results of the MCM uncertainty analysis with $M=50 \times 10^{3}$ simulation events are summarized in Figure 3, with an expected value of $\hat{A}_{0}=$ $1961.062212 \mathrm{~mm}^{2}$, an estimated standard uncertainty of $\pm 0.370792 \mathrm{ppm}$, with an approximate expanded uncertainty of $\pm 0.728266 \mathrm{ppm}$ taking into account a possible double peak. The standard GUM uncertainty analysis however indicates a nominal expected area of $1961.062218 \mathrm{~mm}^{2}$ with a standard uncertainty of $\pm 1.726804 \mathrm{ppm}$ and an expanded uncertainty of $\pm 3.453608 \mathrm{ppm}$ using a coverage factor of $K=2$.

In the final results for the GUM expanded uncertainty we assume an approximate coverage factor of $K=2$ for 
a $95 \%$ confidence interval due to the subjectivity of interpreting what correlation effects to incorporate, and for the MCM expanded uncertainty (in ppm) we estimate the expanded uncertainty by averaging the differences of the lower and upper limits at $95 \%$ confidence level i.e. $10^{6} \times \frac{1}{2}\left[\left(\hat{A}_{0}-A_{0}^{\min \mid p=0.95}\right)+\left(A_{0}^{\max \mid p=0.95}-\hat{A}_{0}\right] / \hat{A}_{0}\right.$ where $\hat{A}_{0}$ is the expected value based on the underlying $\mathrm{PDF}$ for $A_{0}$ not necessarily equal to the nominal value as discussed in Cox and Harris [11]. Based on the number of simulation events from Figure 3 for a probability of $p=0.95$ i.e. $95 \%$ confidence interval an approximate value for the corresponding $\alpha$-quantile was estimated first with $\alpha^{(\text {sym })}=(1-p) / 2=0.025$ assuming the PDF for $A_{0}$ viz. $g\left(A_{0}\right)$ was symmetric and then the exact value was calculated by determining an $\alpha$ to minimize $\hat{G}^{-1}(p+$ $\alpha)-\hat{G}^{-1}(\alpha)$ with the result $\alpha^{(\text {non-sym })}=0.024698$ indicating that it is safe to assume symmetry for the PDF of $A_{0}$ which the GUM analysis implicitly assumes. The endpoints for the $95 \%$ coverage interval of $A_{0}$ were then calculated as $A_{0} \in[1961.060789,1961.063645] \mathrm{mm}^{2}$.

In general a comparison between the results for a GUM and MCM uncertainty analysis is inexact due to the different assumptions and approximations in both techniques, however we can observe that the expectations for the measurand with both techniques are the same in both cases with negligible discrepancy, but that significant differences do exist in the magnitude of each method uncertainty. The GUM is found to overestimate the standard uncertainty and expanded uncertainty by a similar multiplicative factor when compared to the exact results with the MCM technique. This suggests that a single correction factor may be sufficient to scale and modify the GUM results to more closely 'calibrate' the approximate GUM uncertainty estimate to the exact uncertainty obtained using the MCM.

In order to study in more detail what modifications may be necessary to the GUM uncertainty estimate in order to increase its accuracy so that its value is closer to the correct estimate using the MCM, we construct a merit function $\chi$ which we wish to minimize as

$$
\begin{aligned}
\chi= & u_{G U M}\left(A_{0}\right)-u_{M C M}\left(A_{0}\right) \\
= & {\left[K_{1}+K_{2} u^{2}(r)+K_{3} u^{2}(R)+K_{4} \rho_{1}\right.} \\
& \left.+K_{5} \rho_{2}+K_{6} u(r) u(R) \rho_{3}\right]^{1 / 2}-u_{M C M}\left(A_{0}\right) \\
= & f\left(r, R, \rho_{1}, \rho_{2}, \rho_{3}\right) \\
= & f\left(x_{1}, x_{2}, x_{3}, x_{4}, x_{5}\right)
\end{aligned}
$$

where the following abbreviations are utilized:

$$
\begin{aligned}
K_{1} & =\left(\frac{\partial A_{0}}{\partial r_{0}}\right)^{2} u^{2}\left(r_{0}\right) \\
K_{2} & =\left(\frac{\partial A_{0}}{\partial r}\right)^{2} \\
K_{3} & =\left(\frac{\partial A_{0}}{\partial R}\right)^{2} \\
K_{4} & =2 \frac{\partial A_{0}}{\partial r_{0}} \frac{\partial A_{0}}{\partial r} u\left(r_{0}\right)
\end{aligned}
$$

$$
\begin{aligned}
K_{5} & =2 \frac{\partial A_{0}}{\partial r_{0}} \frac{\partial A_{0}}{\partial R} u\left(r_{0}\right) \\
K_{6} & =2 \frac{\partial A_{0}}{\partial r} \frac{\partial A_{0}}{\partial R} \\
\rho_{1} & =\rho\left(r_{0}, r\right), \rho_{2}=\rho\left(r_{0}, R\right), \rho(r, R) .
\end{aligned}
$$

Subject to the mixed inequality constraints:

$$
\begin{aligned}
r_{\min } & \leq x_{1} \leq r_{\max } \\
R_{\min } & \leq x_{2} \leq R_{\max } \\
-1 \leq x_{i} & \leq 1, i \in[3,4,5] .
\end{aligned}
$$

This is in the form of a constrained optimization problem which in the standard formulation is

$$
\begin{array}{r}
\min _{\mathbf{x}} f(\mathbf{x}) \\
g_{j}(\mathbf{x}) \leq 0, j=1,2, \ldots, m \\
h_{j}(\mathbf{x})=0, j=1,2, \ldots, r .
\end{array}
$$

In order to solve for the optimal choice of the piston and cylinder radii and correlation coefficients used as inputs in the calculation for the effective area uncertainty using the standard GUM uncertainty formulation i.e., the choice of inputs which will yield the optimal uncertainty estimate for the effective area uncertainty closest to the actual effective area uncertainty obtained with an exact MCM uncertainty analysis calculation, one can either transform the merit function into an equivalent system of simultaneous nonlinear equations through an augmented Lagrangian formulation or alternately attempt the application of a sequential quadratic programming (SQP) algorithm.

While the optimal choice of these parameters may be investigated for a particular piston/cylinder unit in order to incorporate the varying geometry profiles along with the correlation coefficients in order to scale/modify the GUM uncertainty estimate to more closely align its value with exact MCM based uncertainty estimate, this approach is not considered beneficial as the optimization solution cannot be generalized for arbitrary piston/cylinder geometry profiles.

\section{Conclusions}

In this paper the current approaches used to apply the GUM and MCM analysis techniques for the effective area uncertainty estimation have been studied and the conclusion is that the results for the standard uncertainties and coverage intervals corresponding to the equivalent expanded uncertainties are inconsistent. This is primarily due to the subjectivity of single piston/cylinder radii values utilized in the GUM formulation which dominate in the contribution of the uncertainty. Correlation effects in the GUM approach using the standard measurand model for effective area uncertainty is found to be negligible in the case of distance/radii measurements and inappropriate for piston/cylinder radii correlations.

The MCM uncertainty estimate for $u\left(A_{0}\right)$ is found to be significantly smaller than the GUM estimate although 
it has been determined that both GUM and MCM uncertainties scale approximately the same when standard and expanded uncertainties are considered. However due to the difficulties in further analyzing the effect of radii profiles within the framework of the GUM it is not considered possible to easily calibrate the GUM uncertainty estimate to correspond with the MCM uncertainty estimate.

As a result, we conclude that it is more advantageous to adopt a MCM uncertainty analysis approach as this can very easily incorporate both varying piston/cylinder radii profiles along the engagement length and any applicable correlation effects without constructing elaborate and artificial mechanisms to incorporate similar effects with a GUM framework.

Acknowledgements. This work was performed with financial support by the South African Department of Trade and Industry.

\section{References}

1. R.S. Dadson, S.L. Lewis, G.N. Peggs, The Pressure Balance: Theory and Practice. HMSO (London, 1982)

2. G.N. Peggs, A Method for Computing the Effective Area of a Pressure Balance from Diametral Measurements, Technical Report (NPL, UK, 1977), MOM 23

3. N.D. Samaan, Mathematical Modeling of Instruments for Pressure Metrology, Ph.D. thesis, School of Engineering, City University of London, 1990
4. BIPM, IEC, IFCC, ISO, IUPAC, and OIML, Guide to the Expression of Uncertainty in Measurement. BIPM, 2nd edn. (1995), ISBN 92-67-10188-9

5. JCGM/WG1, Evaluation of Measurement Data - Supplement 1 to the Guide to the Expression of Uncertainty in Measurement - Propagation of Distributions using a Monte Carlo Method. Joint Committee for Guides in Metrology, 1st edn. (2007)

6. M.G. Cox, P.M. Harris, Software Support for Metrology Best Practice Guide No. 6 - Uncertainty Evaluation, Technical Report (NPL, UK, 2004), ISSN 1471-4124

7. G. Buonanno, G. Ficco, G. Giovinco, G. Molinar, Ten Years of Experience in Modeling Pressure Balances in Liquid Media up to Few GPa (Universita degli Studi di Cassino, 2007), ISBN 978-88-8317-037-9

8. R.L. Burden, J.D. Faires, Numerical Analysis (Brooks/Cole, 2001), ISBN 0-534-38216-9

9. R. Willink, A Generalization of the Welch-Satterthwaite Formula for Use with Correlated Uncertainty Components, Metrologia 44, 340 (2007)

10. G. Molinar, M. Bergoglio, W. Sabuga, P. Otal, G. Ayildiz, Calculation of Effective Area $A_{0}$ for Six Pistoncylinder Assemblies of Pressure Balances. Results of the EUROMET Project 740, Metrologia 42, S197 (2005)

11. M.G. Cox, P.M. Harris, Software Specifications for Uncertainty Evaluation. Technical Report (NPL, UK, 2006), ISSN 1744-475

12. R.R. Cordero, G. Seckmeyer, D. Pissulla, F. Labbe, Uncertainty of experimental integrals: application to the UV index calculation, Metrologia 45, 1 (2008) 